\title{
E-cigarette use and COVID-19 in youth and young adults: serious questions about data reliability and call for retraction
}

\author{
Konstantinos Farsalinos ${ }^{1}$, Raymond Niaura ${ }^{2}$ \\ 1 University of West Attica \\ 2 New York University
}

Funding: The author(s) received no specific funding for this work.

Potential competing interests: The author(s) declared that no potential competing interests exist.

\begin{abstract}
In a recent study, Gaiha et al. examined the association between e-cigarette use and COVID-19 in an online crosssectional study of people aged 13-24 years conducted from May 6 to May 14, 2020. We have noticed serious issues in population weighting, response bias and biological implausibility. The suggested conclusions and interpretation of the study findings cannot be considered reliable. These issues raise the question of retracting the study.
\end{abstract}

In a recent study, Gaiha et al. examined the association between e-cigarette use and COVID-19 in an online crosssectional study of people aged 13-24 years conducted from May 6 to May 14, 2020 [1]. The authors found a statistically significant association between ever, but not current, e-cigarette use and COVID-19 diagnosis. They also reported that current e-cigarette use was associated with 2.6-fold higher odds for being tested for COVID-19. A population-weighted proportion of $5.7 \%$ of never and $17.5 \%$ of ever e-cigarette users were reported to have been tested for COVID-19 until May 14, 2020. We have noticed several important problems and implausible findings in this study, which question the reliability of participants' responses, data analysis and overall conclusions. These data quality issues should be evaluated by the authors and the editors of the journal who should consider the option of retracting the publication.

It is not biologically plausible that e-cigarette trial or experimentation would cause health effects that result in stronger predisposition to COVID-19 than current/regular use. Therefore, no causal link between e-cigarette use and COVID-19 can be implied through this finding.

While the high proportion of ever e-cigarette users who were reportedly tested for COVID-19 could explain the high rate of COVID-19 diagnosis, the reliability of participants' responses concerning testing is questionable. According to the CDC, 7,362,526 COVID-19 tests were performed in the US population from March 1 to May 16 (2 days after survey completion) [2]. A weighted proportion of $35.4 \%$ of adolescents aged 13-17 years (data from NYTS 2019) and $25.8 \%$ of Americans aged 18-24 years were ever e-cigarette users [3]. We obtained data about the population size of these age groups from the US Census Bureau (2018) [4], and we estimated that from the 20,818,953 Americans aged 13-17 years and the 30,373,478 Americans aged 18-24 years there are 7,369,909 and 7,836,357 ever e-cigarette users, respectively. The weighted proportions of never and ever e-cigarette users who were tested for COVID-19, as reported by Gaiha et al. (5.7\% and $17.5 \%$, respectively), translate to $4,712,308$ tests performed in age group 13-24 years $(2,661,097$ tests in ever and 
2,051211 tests in never e-cigarette users). This represents $64.0 \%$ of all tests performed in the US until May 16 , a gross overestimation considering the lack of adequate testing capacity at that time and the strong priority given to people at risk for severe COVID-19. US CDC reported that less than 5\% of COVID-19 tests were performed in youth $<18$ years old until May 16 [2]. Thus, the findings by Gaiha et al. are almost certainly based on false-reporting by the participants. Finally, the proportion of participants aged 13-24 years who reported having a diagnosis of COVID-19 would represent $46.8 \%$ of all US confirmed cases until May 14 [5], which is probably another gross overestimation.

In conclusion, the findings by Gaiha et al. cannot be considered valid and population-representative, probably due to serious response bias and the approach of adjusting an online convenience sample to a population-based sample through weighting. The population weighting also seems to be wrong, according to the supplementary table published by the authors, since they used population data from age groups 10-24 years while participants in the study were $\geq 13$ years old. Additionally, the link between ever, but not current, e-cigarette use and COVID-19 suffers from biological implausibility. The authors should reconsider the conclusions and interpretation of their study as presented in the manuscript and the accompanying press release. The problems with data quality raise the issue of retracting the manuscript.

\section{References}

1. Gaiha SM, Cheng J, Halpern-Felsher B. Association Between Youth Smoking, Electronic Cigarette Use, and Coronavirus Disease 2019. J Adolesc Health 2020. doi. 10.1016/j.jadohealth.2020.07.002.

2. Centers for Disease Control and Prevention (CDC). Coronavirus Disease 2019 (COVID-19). COVIDView Summary ending on May 16, 2020. Available at: https:/www.cdc.gov/coronavirus/2019-ncov/covid-data/covidview/pastreports/05222020.html (accessed on August 14, 2020).

3. Centers for Disease Control and Prevention (CDC). Electronic Cigarette Use Among U.S. Adults, 2018. Available at: https://www.cdc.gov/nchs/products/databriefs/db365.htm (accessed on August 14, 2020).

4. United States Census Bureau. Annual Estimates of the Resident Population by Single Year of Age and Sex for the United States: April 1, 2010 to July 1, 2019. Available at: https://www2.census.gov/programs-surveys/popest/technicaldocumentation/file-layouts/2010-2019/nc-est2019-agesex-res.csv (accessed on August 14, 2020).

5. World Health Organization. COVID-19 dashboard. United States of America. Available at: https://covid19.who.int/region/amro/country/us (accessed on August 24, 2020). 